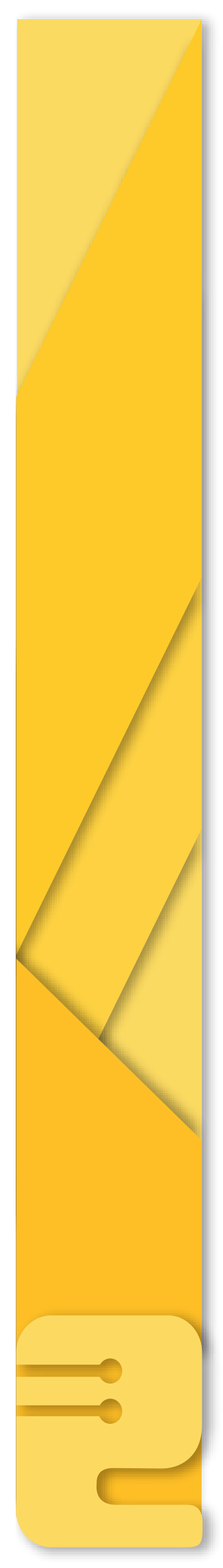

\title{
IMPLEMENTACIÓN DE VOICE PICKING EN TAREAS DE ALISTAMIENTO DE UN OPERADOR LOGÍSTICO EN COLOMBIA ${ }^{1}$
}

\author{
IMPLEMENTATION OF VOICE PICKING IN PACKING TASKS OF A LOGISTICS OPERATOR IN \\ COLOMBIA \\ IMPLEMENTAÇÃO DE VOICE PICKING EM TAREFAS DE ALISTAMIENTO DE UM OPERADOR \\ LOGÍSTICO EM COLÔMBIA
}

Natalia Andrea Falla Betancur², Mauricio Becerra Fernández ${ }^{3}$

Fecha de recibido: Agosto 05 de 2016 | Fecha de aprobado: Septiembre 22 de 2016

\section{Resumen}

Este artículo presenta un análisis sobre la implementación de tecnologías de alistamiento de pedidos mediante el uso de Voice Picking, basado en un caso de estudio aplicado en un centro de distribución de uno de los principales operadores logísticos en Colombia que, a su vez, es contrastado con un sistema actual de Radiofrecuencia. Mediante la comparación de la tasa de alistamiento y el porcentaje de errores obtenidos por los sistemas de Radiofrecuencia y Voice Picking estudiados, se busca sugerir la tecnología a implementar de tal manera que se logre mejorar el desempeño de los procesos de preparación o picking involucrados.

Palabras clave: Alistamiento, Voice Picking, tecnología en centro de distribución.

1 Reporte de caso.

2 Ingeniera industrial de la Universidad Católica de Colombia. Integrante del Semillero Modelos Aplicados a la Logística y la Producción (ModeLoP), adscrito al Grupo de Investigación en Producción GIP de la Universidad Católica de Colombia. Actualmente se desempeña como ingeniera de logística en Suppla S.A. Correo electrónico: nfalla62@ucatolica.edu.co,natafalla@gmail.com

3 Tecnólogo industrial e ingeniero de producción de la Universidad Distrital Francisco José de Caldas. Obtuvo su título de magister en ingeniería industrial en la Universidad Distrital Francisco José de Caldas. Actualmente es candidato a doctor en modelado en política y gestión pública en la Universidad Jorge Tadeo Lozano de Bogotá, Colombia, y PhD. (c) in Model Based Public Planning, Policy Design and Management de la Universidad de Palermo, Italia. Se desempeña como profesor e investigador en las áreas de logística, producción, modelado y simulación en la Universidad Católica de Colombia. Director del Grupo de Investigación en Producción GIP. Correo electrónico: mbecerra@ucatolica.edu.co, mauriciobecerrafernandez@gmail.com 


\section{Abstract}

This paper presents an analysis about the implementation of packing technologies through the use of Voice Picking, based on a case study employed at a distribution centers in one of the main logistics operators in Colombia. Simultaneously, it is matched with a current Radio Frequency system. By the comparison of packing rate and margin of error obtained by Radio Frequency and Voice Picking systems, the aim is to suggest the implemented technology in such a way that the execution of involved packing processes, can be enhanced.

Keywords: Packing, Voice Picking, technology at distribution center.

\section{Resumo}

Este artigo apresenta uma análise sobre a implementação de tecnologias de alistamiento de pedidos mediante o uso de Voice Picking, baseado em um caso de estudo aplicado num centro de distribuição de um dos principais operadores logísticos em Colômbia que, a sua vez, é contrastado com um sistema atual de Rádio-frequência. Mediante a comparação da taxa de alistamiento e a percentagem de erros obtidos pelos sistemas de Radiofrequência e Voice Picking estudados, procura-se sugerir a tecnologia a implementar de tal maneira que se consiga melhorar o desempenho dos processos de preparação ou picking envolvidos.

Palavras chave: Alistamiento, Voice Picking, tecnologia em centro de distribuição. 


\section{INTRODUCCIÓN}

A través de datos estadísticos presentados por la Organización Mundial del Comercio - omc (2012), se observa que el sector de servicios a nivel mundial (en el que se incluyen los servicios prestados por los operadores logísticos), ha tenido un crecimiento exponencial durante el periodo comprendido entre los años 1980 a 2012.Para el caso de Colombia, se observa un comportamiento similar al global; sin embargo, aún se presenta un mayor volumen de importaciones de servicios frente al volumen de exportaciones (ver figura 1).Este fenómeno puede representar una oportunidad específicamente para el campo de logística de almacenamiento para que implemente herramientas tecnológicas que potencialicen los servicios ofrecidos por estos operadores logísticos nacionales.

Figura 1. Comportamiento de las importaciones y exportaciones de servicios en Colombia (1980 - 2012)

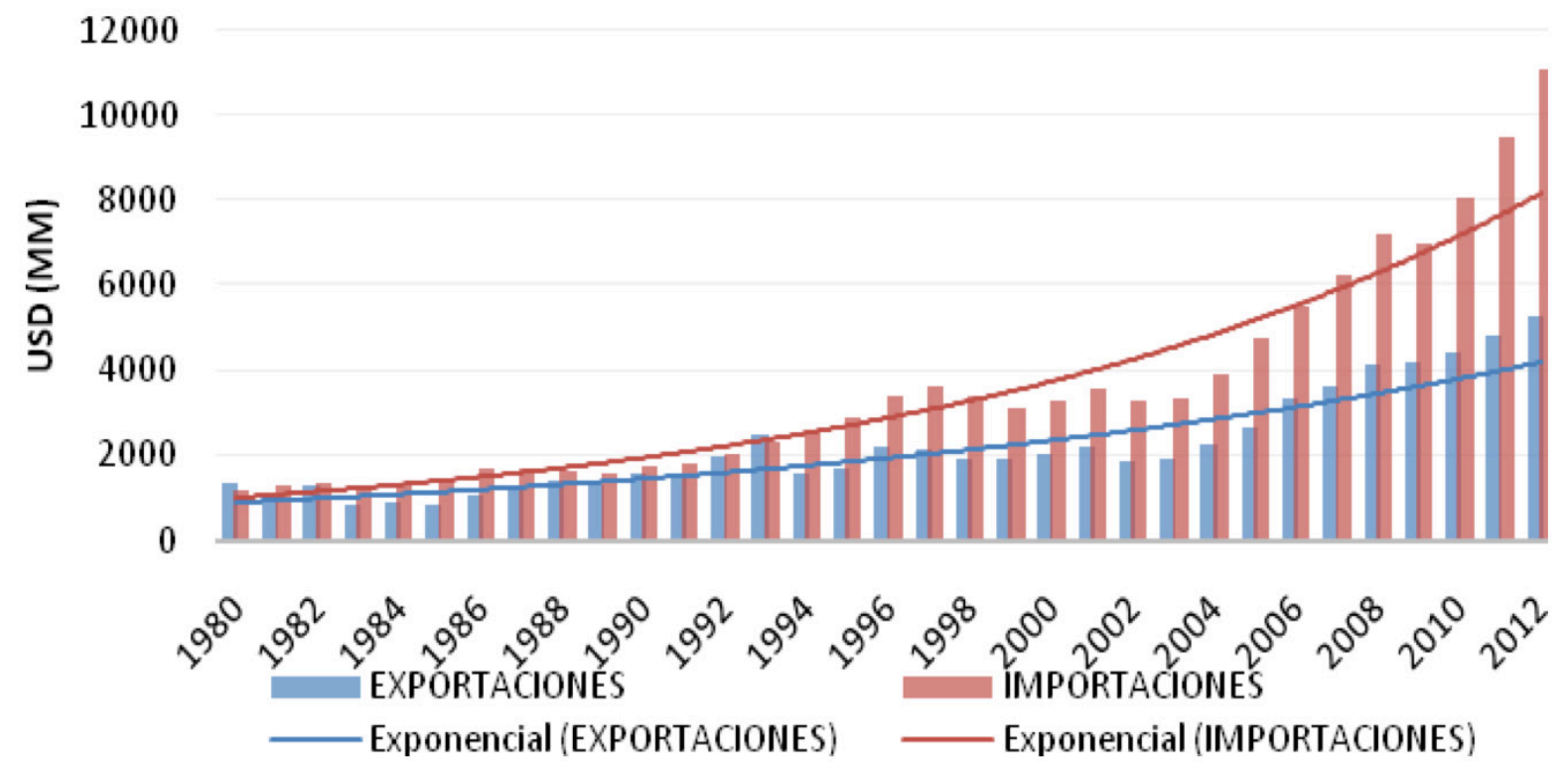

Fuente: OMC, 2012.

El Estado colombiano con el objetivo de facilitar el desarrollo competitivo de las organizaciones logísticas y en coherencia con el comportamiento del mercado internacional y nacional de servicios logísticos, aprobó en el año 2008 el documento conPES No. 3547 del Consejo Nacional de Política Económica y Social, en el que se define la Política Nacional de Logística. En dicho documento se da especial importancia al establecimiento de plataformas logísticas con el propósito de estructurar la cadena de abastecimiento y planificar los flujos de bienes y personas vinculadas a dicha cadena. Por esto, las plataformas e infraestructuras logísticas se convierten en un aspecto clave para impulsar el sector logístico del país.

En el mercado logístico en Colombia se ha identificado la gestión logística como un factor significativo para construir una ventaja competitiva, factor que impacta directamente en la satisfacción y acogida de los clientes. Dentro de dicho campo se resalta la gestión de almacenes como un proceso clave para cumplir con los niveles de servicio, eficiencia y productividad en las operaciones que esta conlleva. Para Espinal, et al. (2010, p.3), las TIC -Tecnologías de la Información y la Comunicación-"se han convertido en un medio para agilizar, flexibilizar y mejorar el intercambio de información y operaciones utilizadas en la gestión de almacenes". En general las Tic son una fuente de desarrollo empresarial en el sector, consideradas como un factor diferenciador desde la perspectiva estratégica de la organización.

Según Mora (2011), las actividades en los centros de distribución, tales como los procesos de recibo, almacenamiento, separación, alistamiento, despacho y manejo de devoluciones de mercancías presentan oportunidades de mejora por medio de lo cual se busca agregar valor a las operaciones relacionadas mediante la aplicación efectiva de herramientas, técnicas, metodologías y nuevos desarrollos que transformen la logística de estos 
centros de distribución en una verdadera ventaja competitiva para las empresas modernas del sector. Estas mejoras configurarían un desempeño eficiente y un aumento del nivel de servicio de cara a los clientes internos y externos.

De acuerdo con Kalenatic, et al. (2009) y Jiménez, et al. (2007), la gestión tecnológica es un proceso de análisis, desarrollo e implementación que le permite a las empresas generar valor económico y obtener una ventaja competitiva a partir de los recursos disponibles, las tecnologías y las tendencias en el mercado al actuar como un agente activo en busca de soluciones tecnológicas para y desde su entorno. Por lo anterior, los sistemas logísticos surgen como un espacio dinámico que permite la gestión tecnológica en las industrias, que desempeñan un papel de soporte para el desarrollo estratégico, soportado en muchos casos en procesos de automatización. Actualmente la automatización de los almacenes se considera una decisión estratégica para la organización, ya que favorece el crecimiento, la reducción de costos operativos y el aumento en la rotación de existencias; sin embargo, la automatización de las operaciones conlleva a la reestructuración de los procesos y los sistemas de soporte que buscarían brindar entregas ágiles y disminuir errores hacia los clientes.

El alistamiento de pedidos es uno de los procesos dentro de un centro de distribución más vulnerables al error humano, debido a que generalmente es donde se presenta mayor intervención manual. Esta preparación de pedidos o picking es el proceso en el cual se recogen productos desde sus lugares de almacenamiento para cumplir con los pedidos de los clientes. Para Yu (2008) se trata de la programación y la liberación de los pedidos de los clientes, la recolección de productos de los lugares de almacenamiento y la eliminación de los elementos seleccionados. Para Bozer y White (1990), el picking es considerado una de las operaciones más costosas del almacén, por esto se presenta una tendencia de la industria por desarrollar tecnología que contribuya a mejorarlo. Adicionalmente, Mauleon (2003) considera que el enfoque de preparación de pedidos busca obtener la coordinación de las estanterías, carretillas, los métodos organizativos, la informática y las nuevas tecnologías para mejorar la productividad, realizar la tarea sin errores y con la calidad requerida por el cliente.

Para López (2010) la preparación de pedidos es la actividad más costosa de las realizadas en el almacén, debido a que comprende alrededor del $65 \%$ del costo de las operaciones. Para Aaron
(2004), esta operación puede llegar a abarcar entre el $40 \%$ y el $60 \%$ del presupuesto de mano de obra de un almacén. Como resultado de esto, la fuerza laboral de una instalación logística y su capacidad para reaccionar positivamente a los cambios en el ambiente de preparación de pedidos, hace que sea un indicador clave de los costos y la productividad.

La inversión en automatización de tareas de alistamiento en dichos CEDI, específicamente en tecnologías de voz -en comparación con otras alternativas-, resulta ser mucho menor y con un retorno de inversión más rápido. Para el caso de estudio de este artículo en un operador logístico colombiano, se cuenta con un área operativa de $12.000 \mathrm{mt}^{2}$ y con un volumen de alistamiento de pedidos hasta un $70 \%$ del total de las operaciones realizadas. Dicho alistamiento es realizado por personal operativo capacitado con terminales de Radiofrecuencia para la atención de la demanda, donde se generan ocasionales errores en el procesamiento de pedidos, aspecto que disminuye el nivel de servicio pactado con el cliente.

Los enfoques sobre la gestión de la manipulación de materiales en los centros de distribución han evolucionado desde los primeros sistemas basados en papel, hasta los sistemas de Radiofrecuencia (RFID) e incluso ambientes informáticos móviles centrados en la voz denominados Voice Picking, por medio de los cuales los encargados del alistamiento pueden comunicarse directamente por voz con el Warehouse Management System (wms), ya que las tareas son transmitidas a través de comandos audibles y confirmadas verbalmente por el operario, lo que deja las manos y el campo visual libre para el desarrollo del alistamiento, según Mora (2011).

Esta tecnología de voz cada día es más demandada por las industrias. En una encuesta realizada por Aberdeen Group (2011) en 134 almacenes de diversas compañías, se determinó que el $94 \%$ de éstos pretenden mejorar o adquirir sistemas dinámicos para sus almacenes; así mismo, se estableció un crecimiento del $44 \%$ en la adquisición de tecnologías de voz en el transcurso del 2011, de los cuales el $20 \%$ adquirieron por primera vez la tecnología y el $22 \%$ la renovaron. Lo anterior refleja un crecimiento en la adquisición de esta tecnología, comparado con los porcentajes de los años anteriores que correspondieron a $17 \%$ en el 2010 y $33 \%$ entre el periodo de 2008 y 2009. Para Mora (2011) uno de los principales beneficios que se puede lograr es que la productividad aumente hasta un 35\% y la precisión hasta un $99 \%$, con una baja formación del operario. 
La incursión de los operadores logísticos en estas tecnologías busca tanto la optimización de tiempos en alistamiento y despacho en función de la fluctuación de la demanda de sus clientes, como la minimización de los costos generados por pedidos no enviados a tiempo, incompletos o errados. Estas tecnologías, entonces, implicarían un aumento en la calidad de sus servicios. Adicionalmente, la tecnología Voice Piking impacta el bienestar laboral, pues se enfoca en mejorar la ergonomía y satisfacción de la mano de obra en el desarrollo de las operaciones de alistamiento.

Para el análisis de la implementación de tecnología Voice Piking, se muestran las etapas del proceso actual de alistamiento con Radiofrecuencia, posteriormente se realizan pruebas tanto con el sistema de Radiofrecuencia como con el sistema Voice Picking. Finalmente se comparan los resultados obtenidos.

\section{MATERIALES Y MÉTODOS}

En el desarrollo de la aplicación tecnológica en el centro de distribución del operador logístico del caso de estudio, se analiza el proceso inicial de alistamiento mediante el uso de Radiofrecuencia y el Sistema de Administración del Almacén (wMS), posteriormente se realiza la implementación y análisis de Voice Picking en dichos procesos de alistamiento, para finalmente contrastar los resultados.

\section{Alistamiento con radiofrecuencia (proceso actual)}

El proceso de alistamiento de pedidos en los primeros niveles de almacenamiento (no se requieren equipos de elevación) mediante el uso de un sistema de información wMS y Radiofrecuencia (RF) se muestra en la figura 2.

Figura 2. Proceso de alistamiento con el uso de Radiofrecuencia

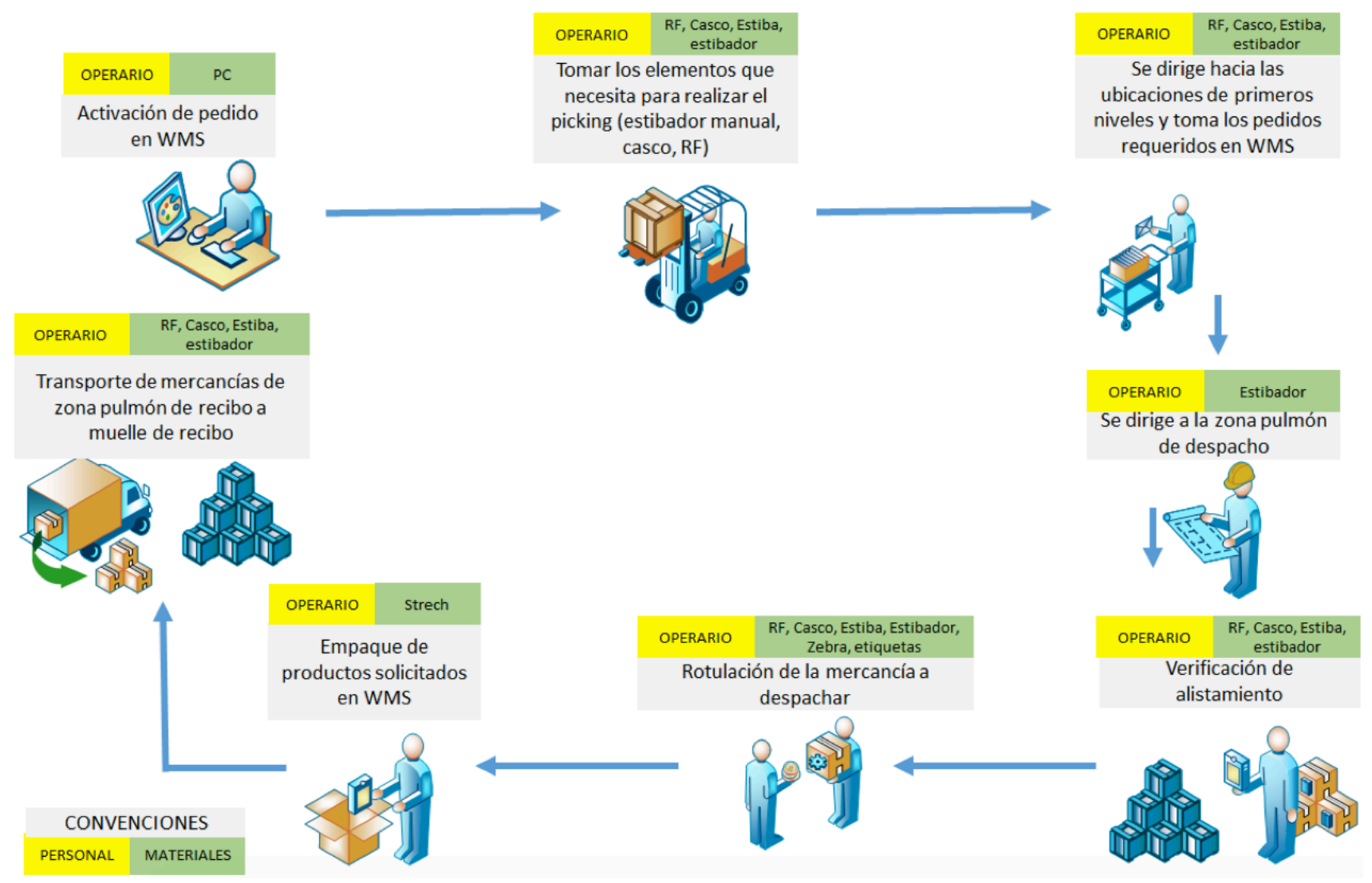

Fuente: elaboración propia. 
Desarrollo de pruebas con los dos tipos de tecnología

En el desarrollo de esta prueba piloto se seleccionó un operario de alistamiento de acuerdo con el criterio del supervisor de bodega del operador logístico que considerará su destreza, habilidad y experiencia en el cargo. La recolección de las muestras se realizó durante 2,5 meses (52 días), tanto para la tecnología actualmente empleada con Radiofrecuencia como para el sistema propuesto de Voice picking en turnos de trabajo de lunes a viernes de las 7:00 a las 17:00 (turno tipo 1) y sábados de las 7:00 a las 10:00 (turno tipo 2).

La principal medida de desempeño en la comparación de las dos tecnologías en los procesos de picking, se basa en el cálculo de la tasa de alistamiento (TA), como se muestra en la ecuación 1:

$$
T A=\frac{C A}{H O}
$$

Donde:

- $T A=$ tasa de alistamiento [cajas/horas]

- $C A=$ número de cajas alistadas

El cálculo de las horas de operación se realiza con base en la ecuación 2 .

$$
H O=(H F-H I) * 24
$$

Donde:

- $H O=$ horas de operación

- $H F=$ hora de finalización del turno

- $H I=$ hora de inicio del turno

\section{RESULTADOS}

El movimiento por tipo de turno de las cajas alistadas y horas en operación mediante el uso del sistema de alistamiento por Radiofrecuencia se muestra en las figuras 3 y 4 .

Figura 3. Cajas alistadas y horas en la operación con el uso de Radiofrecuencia (turno tipo 1)

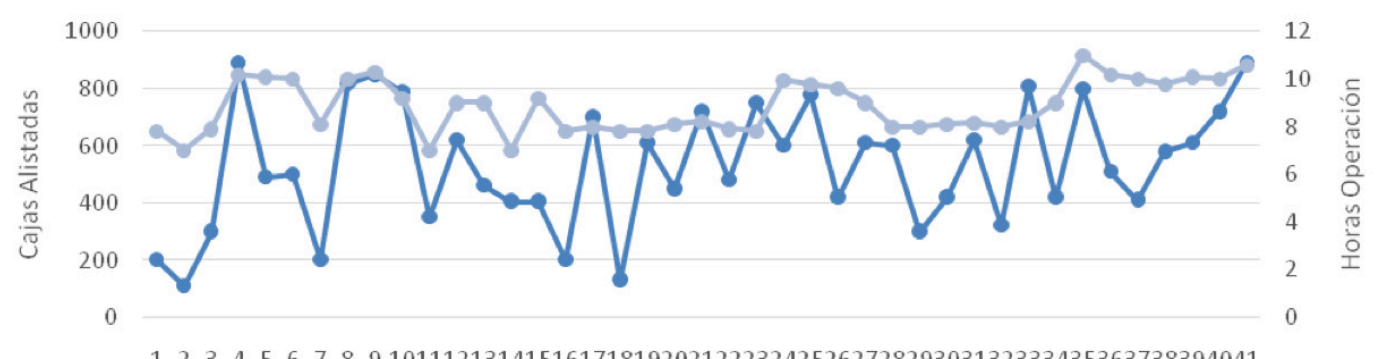

12234567891011121314151617181920212223242526272829303132333435363738394041

Fecha

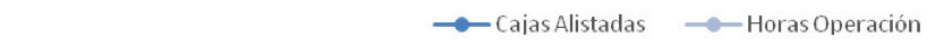

Fuente: elaboración propia.

Figura 4. Cajas alistadas y horas en la operación con el uso de Radiofrecuencia (turno tipo 2)

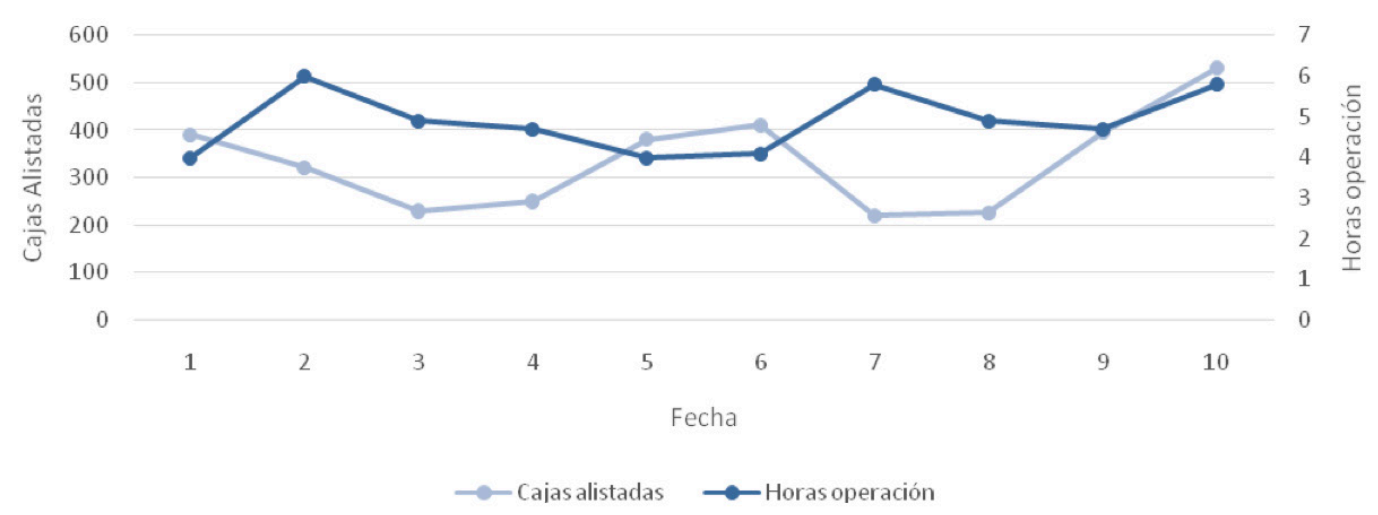


El movimiento por tipo de turno de las cajas alistadas y horas en operación empleando el sistema

de alistamiento por Voice Picking, se muestran en las figuras 5 y 6 .

Figura 5. Cajas alistadas y horas en la operación con el uso de Voice Picking (turno tipo 1)

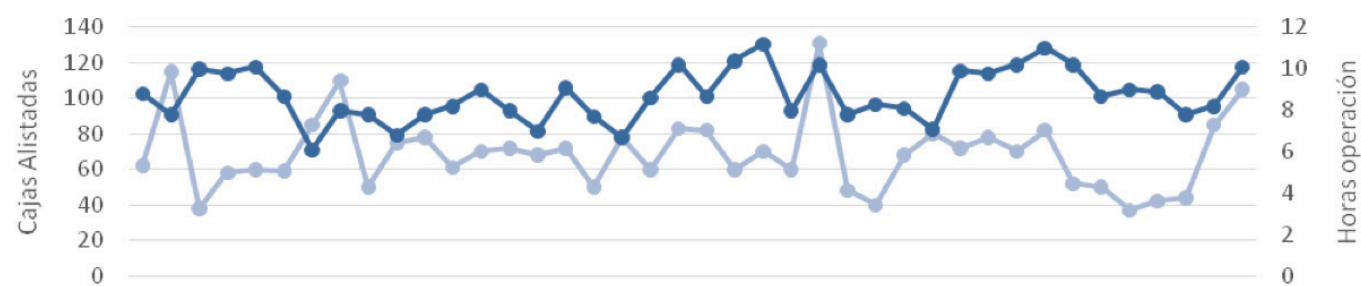

1234556789910111213141516171819202122232425262728293031323334353637383940

$$
\text { Fecha }
$$

$\longrightarrow$ Cajas Alistadas $\rightarrow$ Horas Operación

Fuente: elaboración propia.

Figura 6. Cajas alistadas y horas en la operación empleando Voice Picking (turno tipo 2)

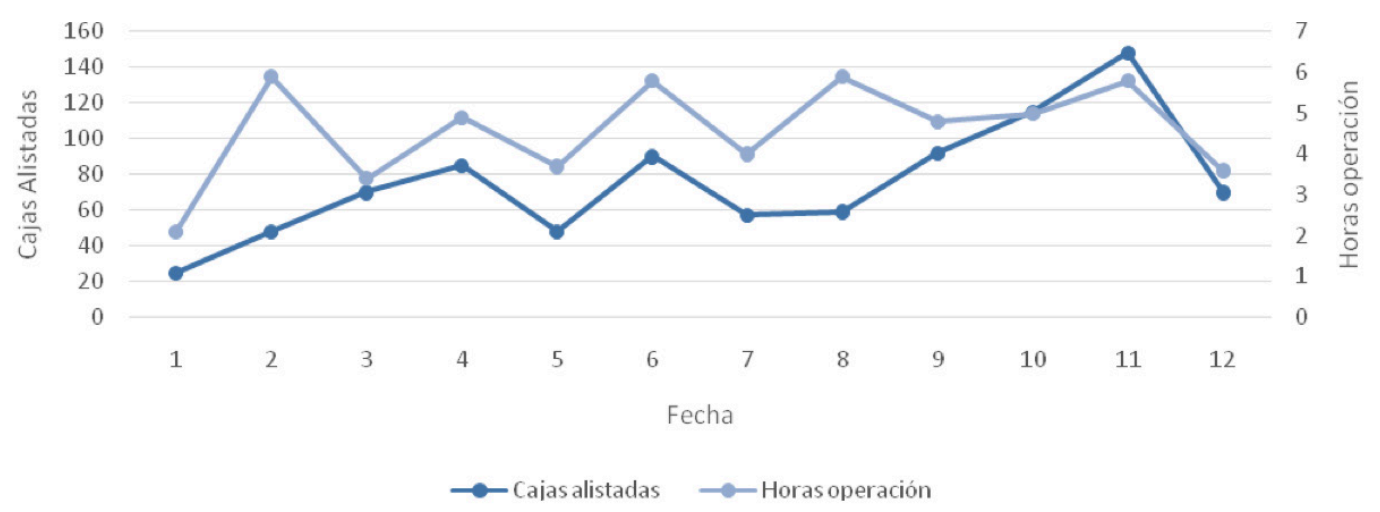

Fuente: elaboración propia.

Los resultados de las principales variables analizadas que comparan las dos tecnologías estudiadas se muestran en la tabla 1:

Tabla 1. Comparativo de resultados

\begin{tabular}{|l|c|c|}
\hline \multicolumn{1}{|c|}{ VARIABLE } & RADIOFRECUENCIA & VOICE PICKING \\
\hline Cajas alistadas & 25.824 & 28.092 \\
\hline Horas de operación & 416,63 & 405,18 \\
\hline Errores en alistamiento de cajas & 28 & 10 \\
\hline Porcentajes de errores de alistamiento & $0,11 \%$ & $0,04 \%$ \\
\hline Tasa de alistamiento (cajas/hora) & 62 & 69 \\
\hline
\end{tabular}

Fuente: elaboración propia. 


\section{CONCLUSIONES}

Mediante la implementación de la tecnología Voice Picking se obtuvo un incremento del $11,86 \%$ en la tasa de alistamiento (se pasó de 62 cajas/hora con Radiofrecuencia a 69 cajas/hora con Voice Picking) y una disminución de errores del 64,29\%(se pasó de 28 errores con Radiofrecuencia a 10 errores con Voice Picking). Lo anterior son algunos indicadores de los aportes que puede tener aplicar este tipo de tecnologías en los procesos de alistamiento.

Si se toma el porcentaje de reducción de errores de la tecnología Voice Picking con respecto al sistema actual de Radiofrecuencia, se puede decir que este último tiene menos posibilidad que el primero de cumplir la fiabilidad del picking acordada con los clientes por medio de Acuerdo de Nivel de Servicio (ANS) que puede llegar a ser del 99,7\%, lo que representa un mínimo de errores. El incumplimiento del Acuerdo puede representar una penalidad hasta del $40 \%$, al tomar como base para su cálculo un mes de margen más los gastos administrativos. Esta penalidad, desde luego, representaría altas pérdidas para el operador logístico.

Según los resultados de la prueba piloto, para lograr los incrementos de productividad señalados por los autores citados (Aaron, López y Mora), es necesario ampliar los periodos de capacitación para lograr la curva de aprendizaje que requiere la nueva tecnología de alistamiento de pedidos.

La aplicación de este tipo de tecnología dentro de los centros de distribución, como se mencionó, se puede extender específicamente a los procesos de recibo e, incluso, a procesos que involucren almacenamiento en logística inversa para proporcionar tanto mayor seguridad en su desarrollo como una mejora del clima laboral. Esto, a su turno, impactaría la rotación del personal y el fortalecimiento de la curva de aprendizaje.

\section{REFERENCIAS}

Aaron, M. (2004). Order Picking for the 21st Century the 21st Century. Raleigh (Carolina del Norte), United States: Tompkins Associates.

Aberdeen Group. (2011). Event Driven Warehousing Trends: Voice Users Speak Out. Boston, Estados Unidos: Aberdeen Group.
Bozer, Y. A., \& White, J. A. (1990). Design and performance models for end-of-aisle order picking systems. Management Science.

Consejo Nacional de Política Económica y Social. (2008). Departamento Nacional de Planeación. Recuperado de: https://colaboracion. dnp.gov.co/CDT/CONPES/Econ\%C3\%B3mi$\cos / 3568$.pdf

Espinal, A., Gomez, R., y Cano, J. (2010). Gestión de Almacenes y Tecnologías de la Información y Comunicación (TIC). Estudios Gerenciales, 26, 117, 45-71.

Jimenez, C. N., Castellanos, O. F., y Morales, M. E. (2007). Tendencias y retos de la gestión tecnológica en economías emergentes. REVISTA Universidad EAFIT, 43 (148), 42-61

Kalenatic, D., González, L., López, C., y Arias, L. (2009). El sistema de gestión tecnológica como parte del sistema logístico en la era del conocimiento. Cuadernos de Administración, 22(39), 257-286. Recuperado de: http://www.redalyc.org/articulo. oa?id=20511993012

López, R. (2010). Logística Comercial. Madrid: Paraninfo.

Mauleon, M. (2003). Sistemas de almacenaje y picking. Madrid: Ediciones Díaz de Santos S.A.

Mora, L. (2011). Gestión logística en centros de distribución, bodegas y almacenes. Bogotá: ECOE Ediciones.

Organización Mundial del Comercio (OMC). (2012). Organización Mundial del trabajo. Recuperado de https://www.wto.org/spanish/ res_s/statis_s/its_s.htm

Yu, M. (2008). Enhancing Warehouse Performance by Efficient [Tesis doctora]. Róterdam, Paises Bajos: Erasmus University. 